\title{
Clopidogrel Resistance is Associated with Long-Term Thrombotic Events in Patients Implanted with Drug-Eluting Stents
}

\author{
Lin Wang, Xiaobei Wang and Fenghua Chen
}

Department of Laboratory Medicine, Union Hospital, Tongji Medical College, Huazhong University of Science and Technology, Wuhan, China

\section{Abstract}

\section{Introduction}

Anti-platelet therapy is the cornerstone management of patients with acute coronary syn-
Background: There are limited prospective data on clopidogrel resistance and clinical outcome of patients with selective coronary drug-eluting stent (DES) implantation.

Objective: To investigate whether clopidogrel resistance is associated with longterm thrombotic events in patients with selective coronary DES implantation.

Methods: A total of 154 patients who underwent selective percutaneous coronary intervention (PCI) with DES were enrolled in this study. Platelet aggregation was measured using light transmittance aggregometry (LTA) before clopidogrel administration (baseline) and 24 hours after loading with clopidogrel $300 \mathrm{mg}$. Clopidogrel resistance was defined as $\leq 10 \%$ absolute difference between baseline aggregation and post-administration aggregation. All patients who received the same anti-platelet treatment were followed up for 1 year after discharge for the incidence of a composite endpoint consisting of cardiovascular death, myocardial infarction (MI) and revascularization, and secondly for the incidence of stent thrombosis.

Results: The incidence of clopidogrel resistance is $20.28 \%$ in our study population. Patients who are complicated by diabetes mellitus, smoke, or have a higher body mass index (BMI) tend to have clopidogrel resistance. Patients in the clopidogrel-resistant group have significantly higher incidences of composite endpoints ( $21.88 \%$ vs $4.92 \%$; $\mathrm{p}=0.006)$ and stent thrombosis $(12.5 \%$ vs $1.64 \%$; $\mathrm{p}=0.017$ ) than patients in the clopidogrel-response group during 1-year follow-up. Conclusions: Diabetes, smoking, and high BMI are associated with clopidogrel resistance, and clopidogrel resistance indicates an increased risk of long-term thrombotic events in patients implanted with DES. 
anti-platelet regimen after drug-eluting stent (DES) implantation for coronary disease. ${ }^{[1]}$ Nevertheless, a large number of cardiovascular events continue to occur despite anti-platelet therapy. Although poor patient compliance can account for some of these events, particularly in those patients who receive DES, increasing evidence indicates that there is variability in response to anti-platelet therapy and patients who have a higher level of platelet reactivity are at an increased risk of recurrent cardiovascular thrombotic events. ${ }^{[2]}$ Therefore, the concept of aspirin and clopidogrel 'resistance' has arisen, and it is indicated that the recurrence of adverse cardiovascular events may be attributed to aspirin or clopidogrel resistance. ${ }^{[3,4]}$ The second-generation P2Y12 blocker clopidogrel whose safety profile is better with a lower incidence of hematologic and liver complications has largely replaced the first-generation P2Y12 blocker ticlopidine in clinical therapy. Several clinical trials have demonstrated that non-responsiveness to clopidogrel is related to a higher risk of cardiovascular events in patients..$^{[5,6]}$ However, little prospective data are available in patients with implantation of DES for longer follow-up periods. In the present study, we aimed to determine clinical factors that were associated with clopidogrel resistance and whether clopidogrel resistance is associated with long-term thrombotic events in patients with selective coronary DES implantation.

\section{Methods}

\section{Study Protocol}

This study was approved by the Ethics Committee of China. 154 patients undergoing selective coronary stent implantation were enrolled after giving written informed consent between January 2006 and January 2008. Exclusion criteria were as follows: (i) history of recent bleeding diathesis or heparin-induced thrombocytopenia; (ii) hematologic or malignant disorder; (iii) serious liver or kidney damage; (iv) serious infection, tumor, or immunologic system disease; (v) cerebrovascular event within the last 3 months; (vi) major surgical operation within 1 week prior; (vii) angiographically visible thrombus in target vessels; and (viii) acute myocardial infarction (MI) within 10 days.

All interventions were performed according to current clinical guidelines, with the implantation of the same type of DES (Partner, Lepu Medical Technology, Beijing, China) and the use of glycoprotein IIb/IIIa inhibitors at discretion of the operator. A weight-adjusted intra-procedural unfractionated heparin (with a goal activated clotting time of 250-300 seconds) was administered during the procedure and was routinely discontinued at the end of the procedure. A $300 \mathrm{mg}$ loading dose of clopidogrel was given to all patients at least 6 hours prior to percutaneous coronary intervention (PCI), followed by a daily dose of $150 \mathrm{mg}$ for 2 weeks and then $75 \mathrm{mg}$ for a minimum of 11.5 months. In addition, from the first day of the procedure, all patients were administered aspirin $300 \mathrm{mg}$ daily for $1 \mathrm{month}$ and then $100 \mathrm{mg}$ for the lifetime of the patient.

\section{Platelet Aggregation}

Blood samples were collected in evacuated containers containing $3.8 \%$ sodium citrate before clopidogrel administration (baseline) and at least 24 (24-28) hours after the loading dose $(300 \mathrm{mg})$ of clopidogrel. Platelet-rich plasma (PRP) was prepared by centrifugation at $1000 \mathrm{~g}$ for 5 minutes and platelet-poor plasma (PPP) was prepared by centrifugation at $4500 \mathrm{~g}$ for 15 minutes. The PRPs were stimulated by adenosine diphosphate (ADP) $20 \mu \mathrm{mol} / \mathrm{L}$. Platelet aggregation was assessed with a Chrono-Log Lume-Aggregometer (model 700) and analyzed using the Aggro/Link software package (Chrono-Log Corporation, Havertown, PA, USA). Clopidogrel resistance was defined as $\leq 10 \%$ absolute difference between baseline aggregation and post-administration aggregation. ${ }^{[7]}$

\section{Clinical Follow-Up and Endpoints}

All patients were followed up and drug therapy compliance was monitored via telephone or an out-patient clinic every month for 1 year after hospital discharge. The two clinical endpoints were as follows: (i) a composite of cardiovascular death, MI, and revascularization; and (ii) definite or probable stent thrombosis. Cardiovascular 
death was defined as death of acute MI, coronary artery disease, or heart failure. The diagnosis of MI was established in the presence of any typical increase or decrease of cardiac biomarkers along with clinical symptoms consistent with cardiac ischemia, following the American College of Cardiology definition. ${ }^{[8]}$ Revascularization includes bypass surgery and PCI. The diagnosis of stent thrombosis was made according to the Academic Research Consortium (ARC) definitions, including definite, probable. or possible. and acute (within 24 hours from procedure), subacute (1-30 days), or late (30 days to 1 year). The determination of definite stent thrombosis required the presence of a coronary syndrome with angiographic or autopsy evidence of thrombus or occlusion. Probable stent thrombosis included unexplained deaths within 1 month after the procedure or acute MI involving the targetvessel territory without angiographic confirmation.

The treating physician and the investigators who evaluated the clinical endpoints were blinded to the results of the platelet aggregation.

\section{Statistical Analysis}

Continuous variables were presented as mean \pm SD and were compared using Student's t-test.
Categorical variables were presented as frequencies and percentages. Patient demographic groups were compared with the chi-squared test, or exact tests if expected cell frequencies were small. Multivariate regression analysis was used to identify if clopidogrel resistance is an independent predictor of thrombotic events. Analysis was performed with SPSS software, version 11.5. All statistical analyses were two-tailed, and a probability value of $p<0.05$ was considered to be statistically significant.

\section{Results}

\section{Basic Characteristics of Study Population}

In the study, a total of 154 patients with the implantation of the same type of DES were enrolled, including 64 females and 90 males. The average age was 60 years (range $30-78$ years). Thirty-two patients $(20.78 \%)$ met the definition of clopidogrel resistance. The characteristics of the study population are presented in table I. Compared with clopidogrel-response patients, clopidogrelresistant patients were more likely to have diabetes mellitus, smoke, and have a higher body mass index (BMI). There were no statistically

Table I. Baseline characteristics of the study population

\begin{tabular}{llll}
\hline & Clopidogrel-resistant group $(\mathrm{n}=32)$ & Clopidogrel-response group $(\mathrm{n}=122)$ & $\mathrm{p}$-Value \\
\hline Mean age \pm SD [y] & $60 \pm 9$ & $60 \pm 10$ & 0.855 \\
Female [n (\%)] & $14(43.75)$ & $50(40.98)$ & 1.000 \\
Dyslipidemia [n (\%)] & $21(65.63)$ & $76(62.30)$ & 0.838 \\
Diabetes mellitus [n (\%)] & $18(56.25)$ & $38(31.15)$ & 0.024 \\
Mean BMI \pm SD & $25.12 \pm 2.61$ & $24.24 \pm 2.11$ & 0.049 \\
Hypertension [n (\%)] & $23(71.88)$ & $84(68.85)$ & 0.831 \\
Smoker [n (\%)] & $15(46.88)$ & $32(26.23)$ & 0.031 \\
Angiographic characteristic [n (\%)] & & & 1.000 \\
$\quad$ one vessel & $8(25)$ & $32(26.23)$ & 0.428 \\
two vessels & $13(40.63)$ & $61(50)$ & 0.259 \\
$\quad$ multiple vessels & $11(34.38)$ & $29(23.77)$ & \\
Medications [n (\%)] & & $107(87.7)$ & 0.767 \\
statins & $29(90.63)$ & $98(80.33)$ & 1.000 \\
$\beta$-blocker & $26(81.25)$ & $115(94.26)$ & 0.241 \\
ACEl or ARB & $28(87.5)$ & $44(36.07)$ & 0.681 \\
glycoprotein Ilb/llIA inhibitor & $10(31.25)$ & & \\
\hline
\end{tabular}

$\boldsymbol{\beta}$-blocker $=\beta$-adrenoceptor antagonists; $\mathbf{A C E I}=$ angiotensin-converting enzyme inhibitor; $\mathbf{A R B}=$ angiotensin receptor blocker; $\mathbf{B M I}=$ body mass index. 
significant differences in the other characteristics between clopidogrel-resistant and clopidogrelresponse patients.

\section{Clopidogrel-Resistant Patients were at Higher Risk of Composite Endpoints and Stent Thrombosis than Clopidogrel-Response Patients}

As shown in table II, composite endpoints occurred in 13 of 154 patients $(8.44 \%)$ during 1 year of follow-up, including four cardiovascular deaths (2.60\%), five MI (3.25\%), and four revascularization $(2.60 \%)$. According to ARC definitions, there were six stent thrombosis in which three were definite $(1.95 \%)$ and three were probable $(1.95 \%)$, and two $(1.30 \%)$ were subacute and four $(2.60 \%)$ were late. No acute stent thrombosis occurred in the patients in this study.

Clopidogrel-resistant patients were at higher risk of composite endpoints and stent thrombosis than clopidogrel-response patients (composite endpoints $21.88 \%$ vs $4.92 \%, \mathrm{p}=0.006$; stent thrombosis $12.5 \%$ vs $1.64 \%, \mathrm{p}=0.017$ ). Although there was no statistical significance in other outcomes, the trend associated with the clopidogrel-resistant group seemed unfavorable.

To examine if clopidogrel resistance is an independent predictor of thrombotic events, we adjusted for diabetes, BMI, and smoking status using multivariate regression analysis. The result indicated that clopidogrel resistance is an independent predictor of composite endpoints and stent thrombosis (composite endpoints $\mathrm{p}=0.008$; stent thrombosis $\mathrm{p}=0.025)$.

\section{Discussion and Conclusions}

Clopidogrel has been regarded as an effective anti-thrombotic drug because it has been shown to yield a $35-60 \%$ reduction in ex vivo platelet aggregation in normal volunteers and patients with stable coronary disease. ${ }^{[4]}$ However, there is individual variability in platelet response to clopidogrel. Thrombotic events still occurred in some patients who were receiving regular clopidogrel therapy and the platelet function of these patients could not be effectively inhibited. This phenomenon is called 'clopidogrel resistance' and the exact mechanism is not yet known. ${ }^{[9]}$ A previous study showed that the rate of clopidogrel resistance in patients ranged between $4 \%$ and $30 \%$, although the definitions of clopidogrel resistance varied. ${ }^{[10]}$ In the present study, the rate of clopidogrel resistance is $20.78 \%$. The first clinical factor we found to be associated with clopidogrel resistance was diabetes, as described in a previous study. ${ }^{[11]}$ Mechanisms responsible for suboptimal clopidogrelinduced anti-platelet effects in diabetic patients include increased exposure to ADP, increased cytosolic levels of calcium, and increased platelet turnover. ${ }^{[12]}$ Furthermore, polymorphism in the

Table II. Incidence of thrombotic events and univariate analysis of clopidogrel response after 12 months

\begin{tabular}{|c|c|c|c|c|}
\hline Event & $\begin{array}{l}\text { Total } \\
(n=154)^{a}\end{array}$ & $\begin{array}{l}\text { Clopidogrel-resistant } \\
\text { group }(n=32)^{\mathrm{a}}\end{array}$ & $\begin{array}{l}\text { Clopidogrel-response } \\
\text { group }(n=122)^{\mathrm{a}}\end{array}$ & p-Value \\
\hline Composite endpoint & $13(8.44)$ & $7(21.88)$ & $6(4.92)$ & 0.006 \\
\hline cardiovascular death & $4(2.60)$ & $2(6.25)$ & $2(1.64)$ & 0.191 \\
\hline MI & $5(3.25)$ & $3(9.38)$ & $2(1.64)$ & 0.061 \\
\hline revascularization & $4(2.60)$ & $2(6.25)$ & $2(1.64)$ & 0.191 \\
\hline Stent thrombosis & $6(3.90)$ & $4(12.5)$ & $2(1.64)$ & 0.017 \\
\hline definite & $3(1.95)$ & $2(6.25)$ & $1(0.82)$ & 0.110 \\
\hline probable & $3(1.95)$ & $2(6.25)$ & $1(0.82)$ & 0.110 \\
\hline \multicolumn{5}{|l|}{ Time of stent thrombosis } \\
\hline acute & 0 & 0 & 0 & \\
\hline subacute & $2(1.30)$ & $1(3.13)$ & $1(0.82)$ & 0.373 \\
\hline late & $4(2.60)$ & $3(9.38)$ & $1(0.82)$ & 0.028 \\
\hline
\end{tabular}


ADP receptor or differences in the post-receptor signaling pathway could not be excluded as an additional explanation for clopidogrel resistance. The second clinical factor we found associated with clopidogrel resistance was smoking and there are no previous data on this relationship. Clopidogrel, a pro-drug, is metabolized in the liver via the cytochrome P450 (CYP) isoenzymes CYP3A4 and CYP1A2, and is converted to the active compound, which inhibits the P2Y12 ADP platelet receptor. Lau et al. ${ }^{[13]}$ have shown that hepatic CYP3A4 activity contributed to clopidogrel resistance. CYP1A2 could be activated by the polycyclic aromatic hydrocarbons that exist in cigarette smoke. ${ }^{[14]}$ Furthermore, nicotine feeding could induce hepatic CYP1A2 in rats. ${ }^{[15]}$ The last clinical factor we found associated with clopidogrel resistance was BMI. It has been reported that elevated BMI is an independent predictor of suboptimal platelet response to clopidogrel in patients undergoing coronary stenting. ${ }^{[16]}$ Previous data also show that obese individuals had an enhanced platelet function and altered metabolism of clopidogrel. ${ }^{[17]}$ The activity of CYP3A4 has been shown to be reduced in overweight individuals. ${ }^{[18]}$ Feher et al. ${ }^{[19]}$ have demonstrated that incidence of clopidogrel resistance is higher in females than in males. Sixty-four women were enrolled in this study and we failed to find a significant difference in the sexes between clopidogrel-resistant and clopidogrel-response groups. Recently, it has been indicated that polymorphism of CYP2C19 is one possible mechanism for clopidogrel resistance. ${ }^{[20-23]}$ CYP2C19 plays a critical role in converting clopidogrel into its active form, and the polymorphism of CYP2C19 is related to the response to clopidogrel and eventually influences the occurrence of thrombotic events after PCI.

The application of DES technology to improve clinical outcomes after PCI represents one of the greatest success stories in cardiology. DES, after coronary angioplasty and bare stent implantation, has been regarded as the third milestone in interventional cardiology. Compared with bare stent, DES can markedly reduce the risk of restenosis. ${ }^{[24]}$ Dual therapy with clopidogrel and aspirin is a standard anti-platelet therapy after DES implantation. Generally, it is recommended that patients with implantation of DES need to take aspirin for the duration of their life and clopidogrel for at least 1 year. Previous studies have shown that clopidogrel resistance was related to the recurrence of cardiovascular events in patients receiving PCI. ${ }^{[25]}$ In the present study, we observed that patients in the clopidogrel-resistant group had a higher risk of composite thrombotic events after DES implantation.

Our study has several limitations as follows: (i) the size of the study population is small; (ii) platelet aggregation was assessed only before and after loading dose, and it is possible that the response to clopidogrel would change during the follow-up; and (iii) aspirin resistance that is associated with clopidogrel resistance ${ }^{[26]}$ was not measured in the study.

In summary, our study showed that diabetes, smoking, and high BMI are associated with clopidogrel resistance in patients implanted with DES. Furthermore, clopidogrel-resistant patients were at a higher risk for composite endpoints and stent stenosis in the 1-year follow-up. Therefore, patients who are likely to have clopidogrel resistance may need platelet monitoring and also require an adjunct anti-thrombotic treatment to adequately inhibit platelet aggregation, especially in a high-risk clinical setting such as PCI.

\section{Acknowledgments}

No sources of funding were used to conduct this study or prepare this article. The authors have no conflicts of interest that are directly relevant to the content of this article.

\section{References}

1. American College of Cardiology, American Heart Association Task Force on Practice Guidelines. 2007 focused update of the ACC/AHA/SCAI 2005 guideline update for percutaneous coronary intervention: a report of the American College of Cardiology/American Heart Association Task Force on Practice Guidelines. Catheter Cardiovasc Interv 2008; 71: E1-40

2. Angiolillo DJ, Fernandez-Ortiz A, Bernardo E, et al. Variability in individual responsiveness to clopidogrel: clinical implications, management, and future perspectives. J Am Coll Cardiol 2007; 49: 1505-16

3. Eikelboom JW, Hirsh J, Weitz JI, et al. Aspirin-resistant thromboxane biosynthesis and the risk of myocardial infarction, stroke, or cardiovascular death in patients at high risk for cardiovascular events. Circulation 2002; 105: 1650-5 
4. Matetzky S, Shenkman B, Guetta V, et al. Clopidogrel resistance is associated with increased risk of recurrent atherothrombotic events in patients with acute myocardial infarction. Circulation 2004; 109: 3171-5

5. Gurbel PA, Bliden KP, Samara W, et al. Clopidogrel effect on platelet reactivity in patients with stent thrombosis: results of the CREST Study. J Am Coll Cardiol 2005; 46: 1827-32

6. Barragan P, Bouvier JL, Roquebert PO, et al. Resistance to thienopyridines: clinical detection of coronary stent thrombosis by monitoring of vasodilator-stimulated phosphoprotein phosphorylation. Catheter Cardiovasc Interv 2003; 59: 295-302

7. Gurbel PA, Bliden KP, Hiatt BL, et al. Clopidogrel for coronary stenting: response variability, drug resistance, and the effect of pretreatment platelet reactivity. Circulation 2003; 107 (23): 2908

8. Cannon CP, Battler A, Brindis RG, et al. American College of Cardiology key data elements and definitions for measuring the clinical management and outcomes of patients with acute coronary syndromes: a report of the American College of Cardiology Task Force on Clinical Data Standards (Acute Coronary Syndromes Writing Committee). J Am Coll Cardiol 2001; 38: 2114-30

9. Angiolillo DJ, Suryadevara S, Capranzano P, et al. Antiplatelet drug response variability and the role of platelet function testing: a practical guide for interventional cardiologists. Catheter Cardiovasc Interv 2009; 73 (1): 1-14

10. Wang TH, Bhatt DL, Topol EJ. Aspirin and clopidogrel resistance: an emerging clinical entity. Eur Heart J 2006; 27: $647-54$

11. Angiolillo DJ, Fernandez-Ortiz A, Bernardo E, et al. Platelet function profiles in patients with type 2 diabetes and coronary artery disease on combined aspirin and clopidogrel treatment. Diabetes 2005; 54: 2430-5

12. Ferroni P, Basili S, Falco A, et al. Platelet activation in type 2 diabetes mellitus. J Thromb Haemost 2004; 2: 1282-91

13. Lau WC, Gurbel PA, Watkins PB, et al. Contribution of hepatic cytochrome P450 3A4 metabolic activity to the phenomenon of clopidogrel resistance. Circulation 2004; 109: 166-71

14. Zevin S, Benowitz NL. Drug interactions with tobacco smoking. Clin Pharmacokinet 1999; 36: 425-38

15. Iba MM, Fung J, Pak YW, et al. Dose-dependent up-regulation of rat pulmonary, renal, and hepatic cytochrome P-450 (CYP) 1A expression by nicotine feeding. Drug Metab Dispos 1999; 27: 977-82
16. Angiolillo DJ, Fernández-Ortiz A, Bernardo E, et al. Platelet aggregation according to body mass index in patients undergoing coronary stenting: should clopidogrel loading-dose be weight adjusted? J Invasive Cardiol 2004; 16: 169-74

17. Scherrer U, Nussberger J, Torriani S, et al. Effect of weight reduction in moderately overweight patients on recorded ambulatory blood pressure and free cytostolic platelet calcium. Circulation 1991; 83: 552-8

18. Kotlyar M, Carson SW. Effects of obesity on the cytochrome P450 enzyme system. Int J Pharmacol Ther 1999; 37: 8-19

19. Feher G, Koltai K, Alkonyi B, et al. Clopidogrel resistance: role of body mass and concomitant medications. Int $\mathrm{J}$ Cardiol 2007; 120 (2): 188-92

20. Simon T, Verstuyft C, Pharm D. Genetic determinants of response to clopidogrel and cardiovascular events. N Engl J Med 2009; 360 (4): 363-75

21. Mega JL, Close SL, Wiviott SD, et al. Cytochrome P2450 polymorphisms and response to clopidogrel. N Engl J Med 2009; 360 (4): 354-62

22. Simon T, Verstuyft C, Mary-Krause M. Genetic determinants of response to clopidogrel and cardiovascular events. N Engl J Med 2009; 360 (4): 363-75

23. Geisler T, Schaeffeler E, Dippon J. CYP2C19 and nongenetic factors predict poor responsiveness to clopidogrel loading dose after coronary stent implantation. Pharmacogenomics 2008; 9 (9): 1251-9

24. Gao RL, Xu B, Chen JL, et al. Immediate and long-term outcomes of drug-eluting stent implantation for unprotected left main coronary artery disease: comparison with baremetal stent implantation. Am Heart J 2008; 155 (3): 553-61

25. Migliorini A, Valenti R, Marcucci R, et al. High residual platelet reactivity after clopidogrel loading and long-term clinical outcome after drug-eluting stenting for unprotected left main coronary disease. Circulation 2009; 120 (22): 2214-21

26. Lev EI, Patel RT, Maresh KJ, et al. Aspirin and clopidogrel drug response in patients undergoing percutaneous coronary intervention: the role of dual drug resistance. J Am Coll Cardiol 2006; 47 (1): 27-33

Correspondence: Lin Wang, MD, Department of Laboratory Medicine, Union Hospital, Tongji Medical College, Huazhong University of Science and Technology, 1277 Jie-Fang Avenue, Wuhan 430022, China. 